\title{
ICDE-Results on Complete Common Cause Failures in the Light of Results Obtained with the POS Model
}

\author{
H.P. Berg, R. Görtz, J. Kesten and L. Weil
}

all Bundesamt für Strahlenschutz (BfS)

\begin{abstract}
Since April 1998, the OECD Nuclear Energy Agency (NEA) is operating the International Common Cause Data Exchange (ICDE) project. For a large number of about 1000 ICDE events the fractions of complete CCFs, which are in most cases of great relevance to bottom line PSA results, have been analyzed as a function of the degree of redundancy. From the point of view of quantitative $\mathrm{CCF}$ modelling, the development of an approach to reflect the empirically available complete CCF fractions in a CCF model application represents a challenging task.

These components are operated under different conditions and technical specifications. Therefore, an approach is chosen which is based on low information distributions of the model parameters to account for the large variation throughout the component populations. The Process Oriented Simulation Model (POS) has been selected to carry out an analysis of complete CCFs. A more than reasonable consistency has been achieved between the model results and the observed results. Moreover, with these sets of distributions as input generic applications of the POS-model become possible. To underline this, generic $\beta$-factors are calculated as an example and compared to generic $\beta$-factors presently used in the design of advanced reactors.
\end{abstract}

\section{INTRODUCTION}

In the frame of Probabilistic Safety Analysis (PSA) for nuclear power plants in many cases Common Cause Failures (CCF) dominate the bottom line results in terms of core damage frequency (CDF) or large release frequency. Dependent failures due to functional dependencies and secondary failures are generally modelled explicitly in a PSA. Other exceeding types of dependent component failures due to systemic or human causes can be introduced into the fault trees by using parametric models. For the CCF evaluation, qualification and quantification a multitude of publications exists based on national and international research and operational experience, see, e.g. [1], [2]. All CCF models need a procedure to adequately estimate the parameters.

Since April 1998, the OECD Nuclear Energy Agency (NEA) is operating the International Common Cause Data Exchange (ICDE) project. The objectives of this exchange comprise, cf. [3], collection and analyses of CCF events, generation of insights into root causes and derivation of preventing approaches, enhancement of experience feedback and, last but not least, facilitation of quantification.

The ICDE-project has been active in communicating key results and features from its large database, for example, on fractions of complete CCFs.

In [4], these data have been interpreted with the POS model. The approach taken is briefly summarized in the following and - subsequently - an improved set of input distributions for generic applications of the POS-model is derived.

\section{THE PROCESS-ORIENTED SIMULATION MODEL (POS)}

The Process Oriented Simulation Model (POS) described in [5], [6] has been selected to carry out a first analytical approach to complete CCFs. The basic idea in the POS modelling approach is to assume some kind of CC impact which, at a given point in time, affects all or a specified subset of the components belonging to the same common cause component group (CCCG). As a consequence, the components affected by the CC impact are assumed either to fail instantly - this is frequently termed a 'shock' - or else delayed. The following sequence of stochastic variables is supposed to adequately describe the CCF process:

- time of CC impact, simulated with a constant CC impact rate $r_{c c}$,

- number of components within the component group of size $r$ affected by the impact,

- times of failure of the impacted components, either immediately or delayed, controlled by model parameter $\mathrm{w}_{\text {inst }}$, the fraction of immediate failures,

- time of detection of the CCF by functional testing.

The probabilities $\mathrm{W}(\mathrm{m}, \mathrm{r})$ for the event that the common cause will affect exactly $\mathrm{m}$ out of $\mathrm{r}$ components are calculated by the following recursive scheme: 


$$
\begin{gathered}
W(2,2)=1 \\
W(3,3)=W(2,2) \bullet F(2,2) \\
W(2,3)=1-W(3,3) \\
W(4,4)=W(3,3) \bullet F(3,3) \\
W(3,4)=W(2,3) \bullet F(2,3)+W(3,3) \bullet(1-F(3,3)) \\
W(2,4)=1-W(3,4)-W(4,4)
\end{gathered}
$$

In these equations, $\mathrm{F}(\mathrm{m}, \mathrm{r})$ is the conditional probability that component $\mathrm{r}+1$ shares the same cause that has been observed in $\mathrm{m}$ out of $\mathrm{r}$ components. Equation (1) reflects the fact that in a system of 2 components both must share the cause. Equations (3) and (6) assure the normalization. Equation (5) reflects that in the system of size $r+1$ that a cause can only be shared by $\mathrm{m}$ components, if in the subsystem of size $\mathrm{r}$ either $\mathrm{m}$ or $\mathrm{m}-1$ components are affected. Equations (2) and (4) are in analogy to (5). The recursive scheme extends to higher degrees of redundancy.

For the conditional probability the following assumption is made, based on observations in simple exactly soluble models:

$$
\begin{aligned}
& F(m, r)=a+b \bullet(m-2) /(r-2) \\
& \mathrm{b}=(1-\mathrm{a}) \bullet\left(1-\exp \left(-\mathrm{r} / \mathrm{r}_{\mathrm{o}}\right)\right)
\end{aligned}
$$

It can easily be verified that a constant $\mathrm{F}(\mathrm{m}, \mathrm{r})$ would lead to results not compatible with operating experience. So equation (7) represents the most simple assumption beyond this. The special choice of $b$ assures that $F(r, r)$ approaches 1 for large $\mathrm{r}$. Simplifying this means that if 9 out of 10 components share the cause it is almost certain that component 10 is affected as well. The two model parameters a and $r_{o}$ offer considerable flexibility for model adjustment to operating experience.

Alltogether, an application of the POS model requires the estimation of the four parameters $r_{c c}, a, r_{o}$ and $w_{\text {inst }}$. Furthermore, the functional test interval is needed.

In many cases, replacing $r_{o}$ by

$$
\mathrm{c}=\exp \left(-1 / \mathrm{r}_{\mathrm{o}}\right)
$$

contributes to simplification of formulae.

\section{APPLICATION OF THE POS MODEL TO ESTIMATE COMPLETE CCF FRACTIONS}

\section{Approach}

In [3], the fractions of complete CCFs have been displayed as a function of the degree of redundancy for a large number of about 1000 ICDE events. Figure 1 shows the results obtained, displayed for degrees of redundancy 2, 3, 4 and 5 or more.

It is important to note that the class of events lumped together to ,5 or more“ does indeed comprise a set of CCF component groups with a widely varying degree of redundancy. This is not reflected in [3] but in the ICDE reports on individual components like [7], [8], [9], [10], [11]. For, e.g. check valves, the range of redundancies goes up to 28 with a major fraction above 10 - one group has even a size of 112 . As an estimate, 10 has been selected as representative of the class with redundancy ,5 or more“ [11].

A large number of events has been collected in the ICDE project for nine different types of components in nuclear power plants from ten different countries. Obviously, these components are operated under different technical specifications and conditions. Therefore, an approach is chosen which is based on low information distributions of the POS model parameters to account for the large variation throughout the component population.

As a first step to provide a rough idea on POS results an extremely "uninformed" point of view is adopted:

- The key model parameters of the POS model $\mathrm{a}, \mathrm{c}$ und $\mathrm{w}_{\text {inst }}$ have a range of possible values from 0 to 1 . It is assumed that the parameters are homogeneously distributed in this full range of possible values.

- As comparison shall be made to the fraction of complete $\mathrm{CCFs}$, the rate of events $r_{\mathrm{cc}}$ is of no influence to the results. 
- For the test interval a homogeneous distribution from 0 to 1 year is assumed. For the class "redundancy 5 and greater" it is assumed that the interval ranges to from 0 to 6 months only, as in the higher redundant systems staggered testing is applied in many cases.

The results of this extremely "uninformed" scenario is displayed in Figure 1. It is not surprising that the resulting fractions are consistently higher than those observed in the ICDE population. The conservative results originate primarily from high values of a and $\mathrm{w}_{\text {inst }}$ and from low values of $\mathrm{c}$.

Fraction of complete CCF

17.04.2007 08:50:49

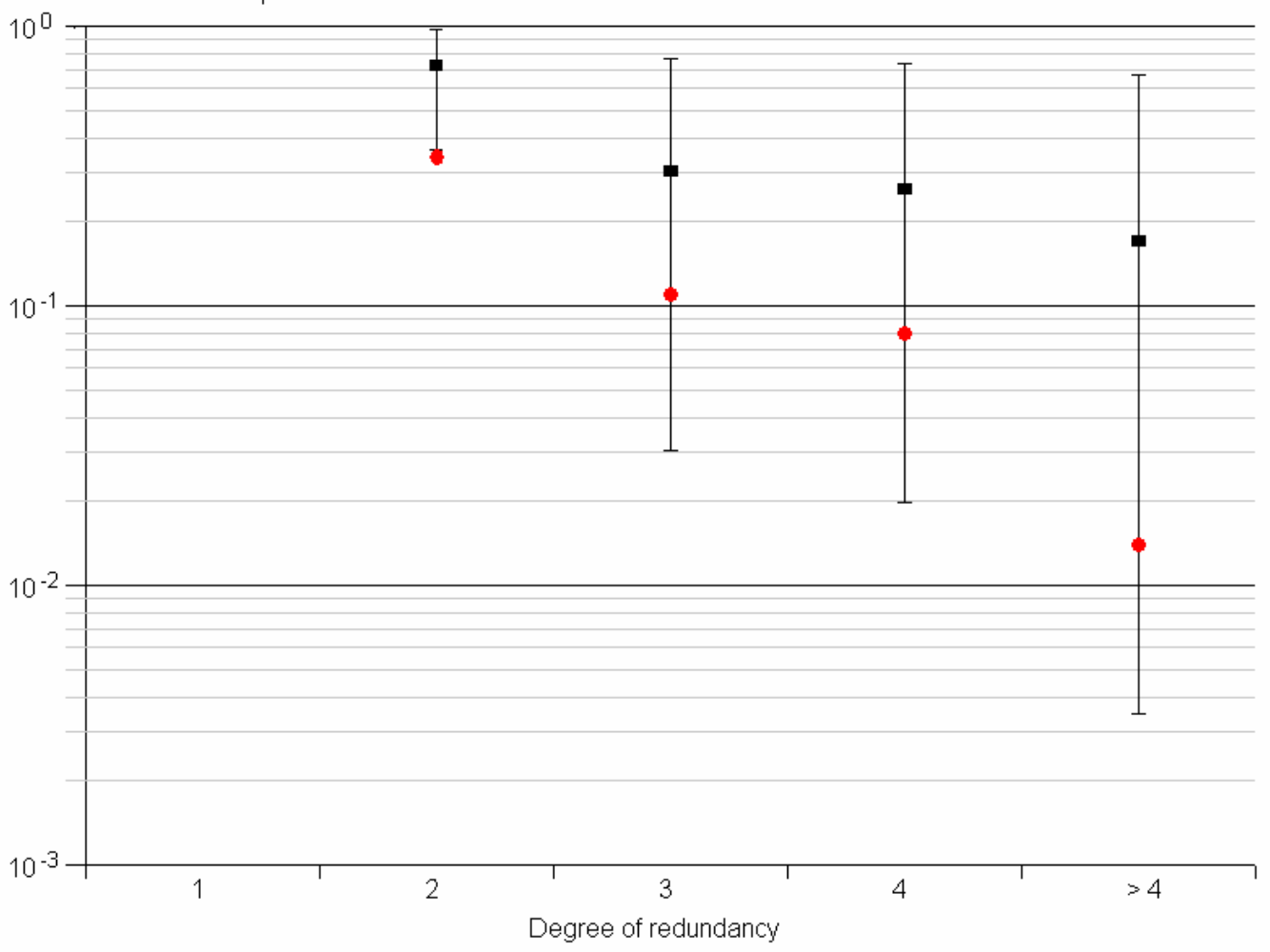

Fig. 1. Fractions of complete CCFs according to the ICDE data base in comparison to those derived from the POS model using extremely "uninformed" input

Circles: Empirical ICDE data

Bar: POS results extending from the 5-percentile over the median (rectangle) to the 95-percentile (median values)

POS input consistent with the ICDE data on complete CCFs

To achieve a set of POS input distributions consistent with the ICDE information displayed in Figure 1, cut-offs are introduced into the parameter distributions in the following manner:

$$
\begin{gathered}
0<\mathrm{a}<0,7 \\
0<\mathrm{w}_{\text {inst }}<0,15 \\
0,5<\mathrm{c}<1
\end{gathered}
$$

There are still considerable parameter ranges, but they have been narrowed down to achieve the complete CCF fractions displayed in Figure 2. Obviously, a good consistency with the observed values has been achieved. 


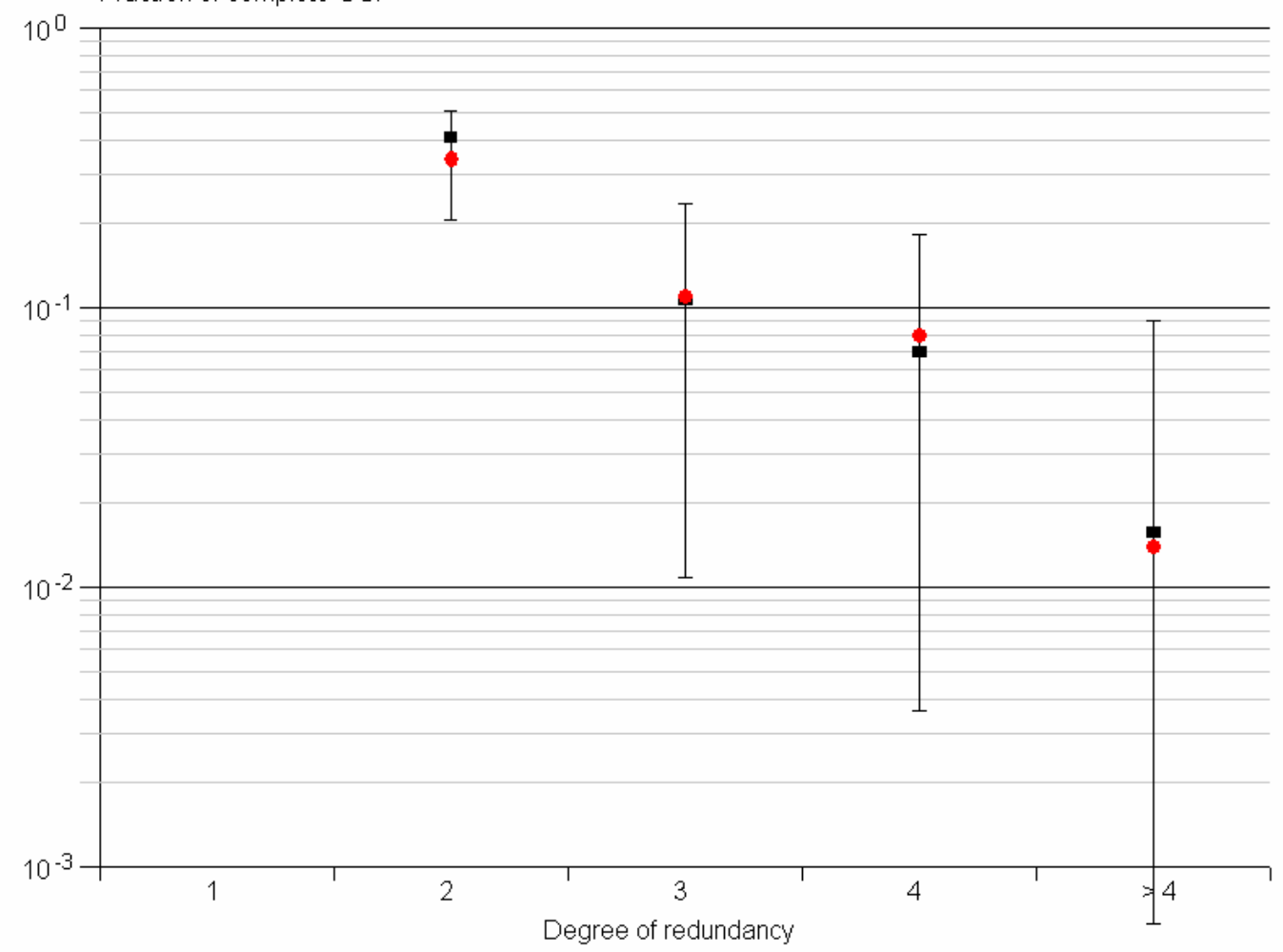

Fig. 2. Fractions of complete CCFs according to POS results fitted to the observed ICDE data Circles: Empirical ICDE data

Bar: POS results extending from the 5-percentile over the median (rectangle) to the 95-percentile (median values)

In all cases the simulations comprised 1000 different sets of parameter values to achieve a sufficient statistical basis for the results.

\section{DERIVATION OF GENERIC BETA-FACTORS WITH THE POS MODEL AND COMPARISON TO PRACTICES IN REACTOR DESIGN}

\section{Use of Generic CCF Data in Reactor Design}

In [12], the approach taken to CCF analysis in the frame of design for the EPR has been described an important part of the quantitative evaluation is the beta-factor model as described in the European Utility Requirements. The key features of this approach can be summarized as follows:

- All components of the CCCG of size $r$ are hit by shocks.

- lethal shocks

- rate $\omega$

- $\quad$ conditional failure probability $=1$

- non-lethal shocks

- rate $\mu$

- $\quad$ conditional failure probability $=\rho$

- $\quad \beta$ - Factors

- total component failure rate

$$
\begin{array}{cc}
\beta(\mathrm{k}, \mathrm{r}) \cdot \lambda=\mu \cdot \rho^{\mathrm{k}} \cdot(1-\rho)^{\mathrm{r}-\mathrm{k}} & \text { if } \mathrm{k}<\mathrm{r} \\
\beta(\mathrm{k}, \mathrm{r}) \cdot \lambda=\mu \cdot \rho^{\mathrm{r}}+\omega & \text { if } \mathrm{k}=\mathrm{r} \\
\lambda=\lambda_{\text {ind }}+\mu \cdot \rho+\omega &
\end{array}
$$


Table 1. $\beta$-Factors as applied in [12]

\begin{tabular}{|c|c|c|c|c|c|c|c|}
\hline $\mathrm{k}{ }^{\mathrm{r}}$ & 2 & 3 & 4 & 5 & 6 & 7 & 8 \\
\hline 2 & 0,05 & 0,03 & 0,02 & 0,01 & 0,009 & 0,006 & 0,004 \\
\hline 3 & - & 0,02 & 0,01 & 0,007 & 0,004 & 0,003 & 0,002 \\
\hline 4 & - & - & 0,01 & 0 & 0 & 0 & 0 \\
\hline 5 & - & - & - & 0,007 & 0 & 0 & 0 \\
\hline 6 & - & - & - & - & 0,006 & 0 & 0 \\
\hline 7 & - & - & - & - & - & 0,005 & 0 \\
\hline 8 & - & - & - & - & - & - & 0,005 \\
\hline
\end{tabular}

The data in table 1 is obtained with the following choice of parameters:

$$
\begin{array}{ll}
- & \rho=1 / 3 \\
- & \mu=0,405 \cdot \lambda \\
- & \omega=0,005 \cdot \lambda
\end{array}
$$

They were derived for specific sets of $\mathrm{k}$ out of $\mathrm{r}$ failures.

The zero figures, however, are not derived from the formulas given above, but have been set. The exact reasons for this artificial model modification and the data base upon which the parameters were estimated are not known to the authors of this paper.

Nevertheless, having derived a generic set of input quantities for the POS model, the question comes up, which results can be obtained for the beta-factors with this tool and which insights can be obtained by comparing the two approaches.

\section{Beta-factors obtained with the POS model}

In this section, the POS model is applied to calculate beta-factors with the generic input distributions that have been obtained in chapter 3 from the ICDE data on complete CCF. The $\beta$-factors are derived directly from formulae (13) and (14) using as input the conditional failure probabilities obtained with the POS model. As the $\beta$-factors displayed in table 1 are defined for sets of specific components the POS results must be adjusted by division through the combinatorial factor. The results are shown in table 2 .

Table 2. $\beta$-Factors calculated with the POS-model using the parameter distributions according to Fig. 2 (median values)

\begin{tabular}{|c|c|c|c|c|c|c|c|}
\hline $\mathrm{k}{ }^{\mathrm{r}}$ & 2 & 3 & 4 & 5 & 6 & 7 & 8 \\
\hline 2 & 0,20 & 0,05 & 0,02 & 0,008 & 0,005 & 0,003 & 0,002 \\
\hline 3 & - & 0,06 & 0,01 & 0,005 & 0,002 & 0,001 & 0,0006 \\
\hline 4 & - & - & 0,03 & 0,007 & 0,002 & 0,0009 & 0,0004 \\
\hline 5 & - & - & - & 0,02 & 0,004 & 0,001 & 0,0004 \\
\hline 6 & - & - & - & - & 0,02 & 0,003 & 0,0008 \\
\hline 7 & - & - & - & - & - & 0,02 & 0,002 \\
\hline 8 & - & - & - & - & - & - & 0,01 \\
\hline
\end{tabular}


The comparison between the $\beta$-factors from the BFR model in table 1 and those obtained with the POS model with ICDE consistent input distributions shows some agreement, but especially for higher failure multiplicities a clear tendency of higher values in table 2 is observed, typically by about a factor of 3 .

The question must be asked whether this discrepancy is due to the different sets of events - in the first case from French PSA and in the ICDE from many countries - or due to the different modelling approaches. The answer is very clearly that the different sets of events are the prime cause. This can easily be seen be noting that the 4 out of 4 fraction according to table 1 and formulae (13) and (14) would be about 0,025 , which is by a factor 3 lower than the corresponding empirical value from ICDE. It can therefore be expected that a corresponding modification of the POS input distributions would bring the two approaches much closer together. By simply reducing the cut-off for parameter a from 0.7 to 0.35 , a reduction of the 4 out of 4 fraction calculated with the POS model is achieved. In table 3 , the corresponding $\beta$-factors are given. The deviations from the values in table 1 are indeed much less significant.

Table 3. $\beta$-Factors calculated with the POS-model using the parameter distributions according to Fig. 2 but a cut-off for parameter a of 0,35 (median values)

\begin{tabular}{|c|c|c|c|c|c|c|c|}
\hline $\mathrm{k}^{\mathrm{r}}$ & 2 & 3 & 4 & 5 & 6 & 7 & 8 \\
\hline 2 & 0,2 & 0,06 & 0,03 & 0,01 & 0,007 & 0,004 & 0,003 \\
\hline 3 & & 0,03 & 0,009 & 0,004 & 0,002 & 0,001 & 0,0007 \\
\hline 4 & & & 0,01 & 0,003 & 0,001 & 0,0007 & 0,0004 \\
\hline 5 & & & & 0,008 & 0,001 & 0,0006 & 0,0003 \\
\hline 6 & & & & & 0,006 & 0,001 & 0,0003 \\
\hline 7 & & & & & & 0,005 & 0,0007 \\
\hline 8 & & & & & & & 0,004 \\
\hline
\end{tabular}

There are, however, still some differences for higher redundancies. These are mainly due to the different nature of the BFR and the POS models. The BFR model produces a strong decrease of values with increasing failure multiplicity. In table 1 , the values from 4 out of 8 failures on have been set to zero, indicating a limited trust in the model results in this range. Finally, for the complete failure 8 out of 8 the value resulting from the lethal-shock assumption is obtained. It is interesting to note that the POS results in table 3 decrease as those of the BFR model, but then increase toward the complete failure. This increase is smooth and does not imply such a strong and hardly credible variation as in the BFR model.

The discrepancy for $\beta(2,2)$ amounts to a factor of 4 and is not removed with the reduced cut-off in parameter a. It is mainly due to different normalization. The BFR model with the data given above implies a conditional probability of a non-lethal shock with no failures at all of 0,49. As it is not clear whether or not - and if, to what degree - these events would be identified as common cause events they are not considered in the POS approach. If these events were removed from the BFR model the different normalization would lead to an enhanced $\beta(2,2)$ by about a factor of 2 and thus the discrepancy would be reduced.

The simulation model provides in addition to the median values given in the tables above, additional data characterizing the distribution of the $\beta$-factors. Examples are given in table 4 . The distributions of the $\beta$-factors are obviously not lognormal.

Table 4. Example for $\beta$-Factor uncertainly characteristics, POS input distributions as those used for table 2 (according to (10), (11) and (12))

\begin{tabular}{|c|c|c|c|c|}
\hline \multirow{2}{*}{$\mathrm{k}$} & \multicolumn{4}{|c|}{$\beta(\mathrm{k}, 4)$} \\
\cline { 2 - 5 } & \multirow{2}{*}{ mean } & 5 & 50 & 95 \\
\cline { 3 - 5 } & $1,810^{-2}$ & $1,110^{-2}$ & $1,710^{-2}$ & $6,310^{-2}$ \\
\hline 2 & $1,210^{-2}$ & $1,910^{-3}$ & $1,310^{-2}$ & $1,810^{-2}$ \\
\hline 3 & $3,410^{-2}$ & $2,010^{-3}$ & $3,210^{-2}$ & $7,610^{-2}$ \\
\hline
\end{tabular}




\section{SUMMARY, CONCLUSIONS AND OUTLOOK}

OECD/NEA`s ICDE project has brought and will continue to bring insights into common cause failures - in many cases key contributers to bottom line PSA results - based on an international exchange with broad participation. Important results are shared by the participants with the international community. As an example, for around $1000 \mathrm{CCF}-$ events the fractions of complete CCFs have been published.

Two conclusions can be drawn from the exercise carried out in this paper. Firstly, the comparison of POS results obtained from very broad distributions - corresponding to the large variations in design, operation and maintenance of the CCF component groups from the large ICDE component group population - of the model parameters in a first step led to rather uncertain results, which overestimated the empirical data, which were lying in the uncertainty bands. A very consistent picture can be obtained by introducing cut-offs into the model parameter distributions.

The comparison of $\beta$-factors applied in reactor design [12] with those obtained with the POS model using the input distributions fitted to the ICDE data on complete CCF brings a clear tendency of higher values on the POS/ICDE side. It can be shown that is primarily due to differences in the sets of CCF events in both approaches. Some of the deviations, however, are rooted in differences between the two models. The POS model seems to offer more credible results for highly redundant systems.

It would be interesting to have similar approaches based on different CCF models to derive complete CCF fractions, for comparison.

Another essential point to be made is that the POS approach presented here is largely based on published material.

A BfS-report is in preparation, which summarizes the POS model development, its features and the applications carried out up to now.

\section{REFERENCES}

1. Mosleh, A., Rasmuson, D.M., Marshall, F.M., Guidelines on Modeling Common-Cause Failures in Probabilistic Risk Assessment, NUREG/CR-5485, November 1998.

2. Mosleh, A., Rasmuson, D.M., Marshall, F.M., Common-Cause Failure Parameter Estimation, NUREG/CR-5497, October 1998.

3. Johanson, G., Kreuser, A., Pyy, P., Rasmuson, D., and Werner, W., „OECD/NEA International Common Cause Data Exchange (ICDE) Project - insights and lessons learned,“ Kerntechnik 71, 1-2, p 13, Carl-Hanser Verlag, München

4. Görtz, R., Berg, H.P., Fröhmel, T., Kesten, J., Weil, L. „Calculating Generic B-Factors for Common Cause Failure Analysis with the POS Model“", Jahrestagung Kerntechnik, Karlsruhe, Mai 2007

5. Berg, H.-P., Görtz, R., and Schimetschka, E.: A Process Oriented Simulation Model for Common Cause Failures. Kerntechnik 67, 2-3, p 72, Carl-Hanser Verlag, München (2002)

6. Berg, H.P., Görtz, R., and Schimetschka, E., „A Model for Common Cause Failures in Systems of Redundant Components and Applications", Proc. of the $8^{\text {th }}$ Internat. Conf. on Probabilistic Safety Assessment and Management PSAM '8, New Orleans, May 23 - 27, 2006

7. NEA/CSNI/R(99) 2. ICDE Project Report on Collection and Analysis of Common-Cause Failures of Centrifugal Pumps. Eds.: Werner, W. ; Johanson, G. February 2000

8. NEA/CSNI/R(2000) 20. ICDE Project Report on Collection and Analysis of Common-Cause Failures of Emergency Diesel Generators. Eds.: Wierman, T.E. ; Rasmuson, D.M.;Marshall, F.M. February 2001

9. NEA/CSNI/R(2001) 10. ICDE Project Report on Collection and Analysis of Common-Cause Failures of Motor Operated Valves. Eds.: Kreuser, A. ; Schulze, V.; and Tirira, J. July 2001

10. NEA/CSNI/R(2002) 19. ICDE Project Report on Collection and Analysis of Common-Cause Failures of Safety and Relief Valves. Eds.: Jonsson, E.; Johanson, G.;Pesonen, J.; Jänkällä, K.; Werner, W. October 2002

11. EA/CSNI/R(2003) 19. ICDE Project Report on Collection and Analysis of Common-Cause Failures of Check Valves. Eds.: Theiss, K.; Hessell, P.; Werner, W. October 2002

12. Blombach, J., Bordihn, S., Kollasko, H., „CCF analysis for new reactor designs“, Kerntechnik 71, 1-2, p 29, CarlHanser Verlag, München 